\title{
Selection of Structure-Switching Aptamer for the Specific Methotrexate Detection
}

Junqing $\mathrm{He}^{1}$, Junyan Wang ${ }^{2}$, Min Zhang ${ }^{1 *}$, Guoyue $\mathrm{Shi}^{1 *}$

${ }^{1}$ School of Chemistry and Molecular Engineering, Shanghai Key Laboratory for Urban Ecological Processes and Eco-Restoration, Research Center of Nanophotonics and Advanced Instrument, Ministry of Education, East China Normal University, Dongchuan Road 500, Shanghai 200241, China

${ }^{2}$ Institute of Molecular Medicine, Renji Hospital, School of Medicine, Shanghai Jiao Tong University, Shanghai, China

* Corresponding author: Guoyue Shi (gyshi@chem.ecnu.edu.cn), Min Zhang (mzhang@chem.ecnu.edu.cn)

\section{Table of Contents}

Figure S1. Structure of MTX and other small molecules used in this work.

Figure S2. CD spectra of MTX, HMX38 and MTX + HMX38.

Figure S3. Fluorescence intensity of G12T, G16T and SLAT with different concentration of MTX utilizing intermolecular strand displacement assay.

Figure S4. Optimization of 38-3 biosensor concentration.

Figure S5. Optimization of 38-3 biosensor reaction temperature.

Figure S6. Fluorescence intensity of different concentration serum with $480 \mathrm{~nm}$ excitation, 520 $\mathrm{nm}$ emission and $630 \mathrm{~nm}$ excitation, $668 \mathrm{~nm}$ emission.

Table S1. Comparison of previously reported methods for MTX detection.

Table S2. Selection protocol of a successful MTX C-SELEX experiment.

Table S3. DNA sequences used in C-SELEX.

Table S4. Sequencing results of a final library pool from MTX aptamer selection.

Table S5. DNA sequences used in ITC.

Table S6. DNA sequences of intramolecular strand-displacement fluorescence sensors.

Table S7. DNA sequences of intermolecular strand-displacement fluorescence sensors. 


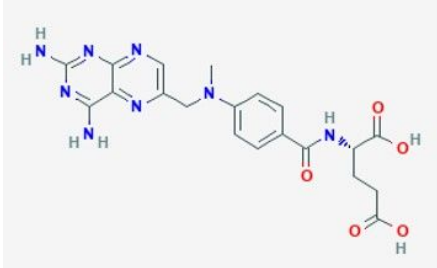

Methotrexate

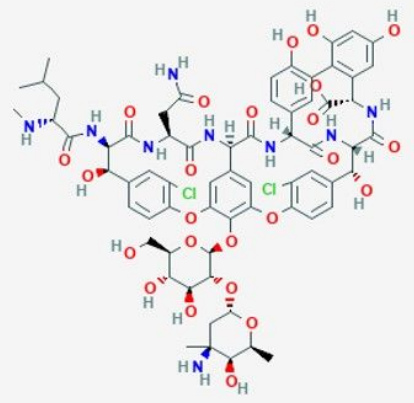

Vancomycin

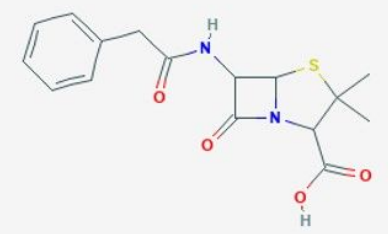

Penicillin

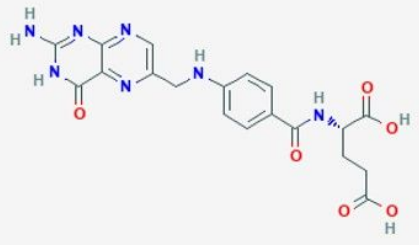

Folic acid

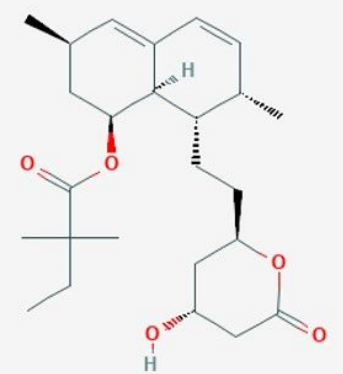

Simvastatin

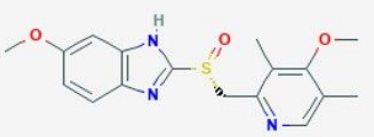

Omeprazole

Fig. S1. Structure of methotrexate and other small molecules used in this work.

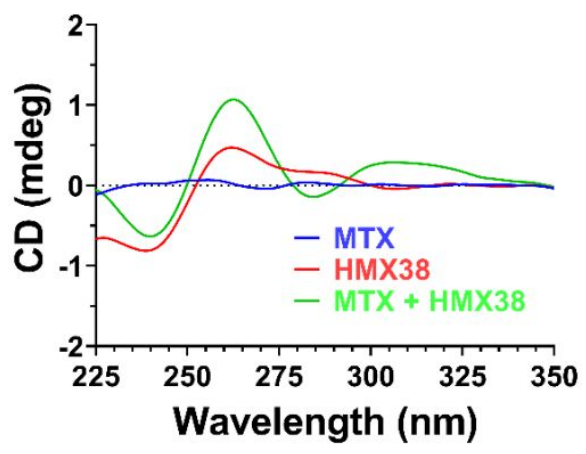

Fig. S2. CD spectra of MTX, HMX38 and MTX + HMX38. 

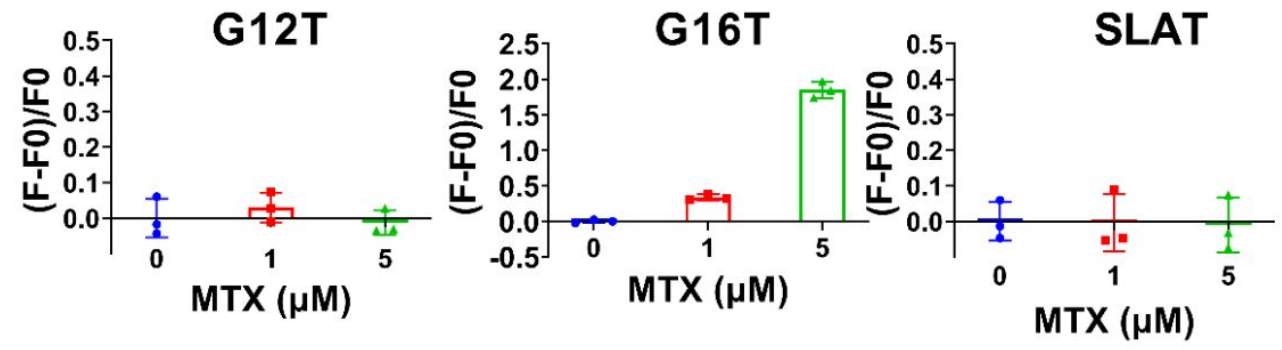

Fig. S3. Fluorescence intensity of G12T, G16T and SLAT with different concentration of MTX utilizing intermolecular strand displacement assay.

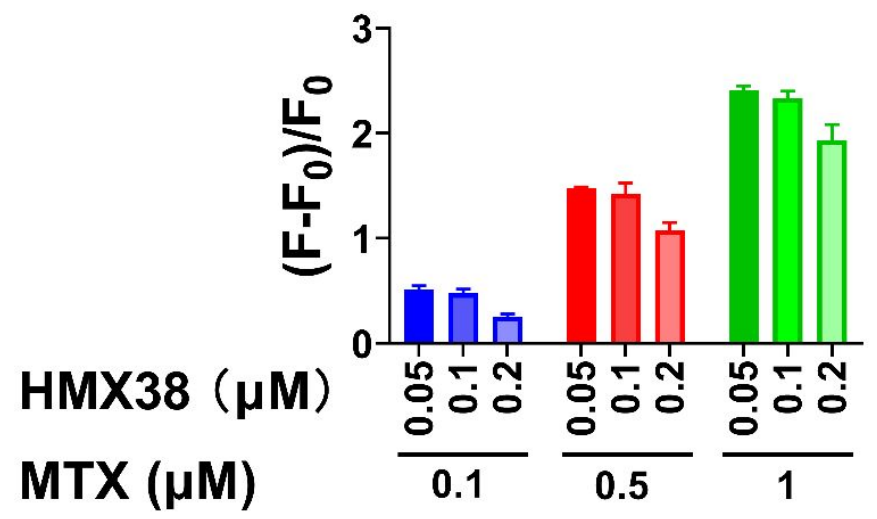

Fig. S4. Optimization of 38-3 biosensor concentration.

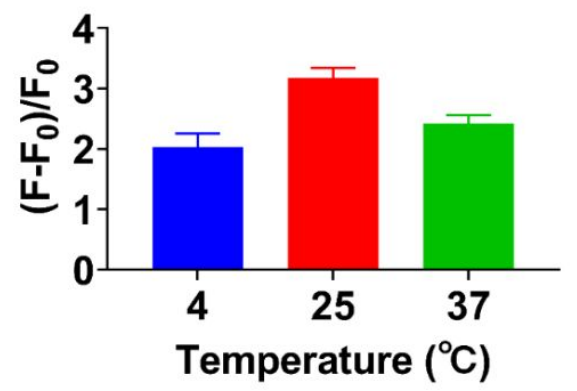

Fig. S5. Optimization of 38-3 biosensor reaction temperature.

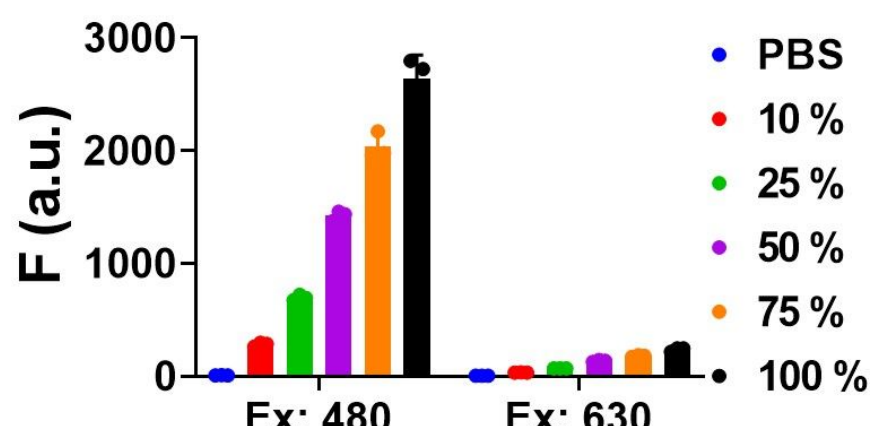

Fig. S6. Fluorescence intensity of different concentration serum with $480 \mathrm{~nm}$ excitation, $520 \mathrm{~nm}$ emission and $630 \mathrm{~nm}$ excitation, $668 \mathrm{~nm}$ emission. 
Table S1. Comparison of previously reported methods for MTX detection

\begin{tabular}{lllllll}
\hline Method & Time & Sample & Cost & LOD & Linear range & Cited Reference \\
\hline HPLC & +++ & Serum & +++ & $0.003 \mu \mathrm{M}$ & $0.025-5.00 \mu \mathrm{M}$ & 1 \\
FPIA & +++ & Serum & +++ & $/$ & $20-1000 \mathrm{nM}$ & 2 \\
LC/MS-MS & +++ & Serum & +++ & $3 \mathrm{nM}$ & $10-1000 \mathrm{nM}$ & 3 \\
EMIT & +++ & Serum & ++ & $0.05 \mu \mathrm{M}$ & $0.05-1.00 \mu \mathrm{M}$ & 4 \\
SPR & + & Serum & + & $28 \mathrm{nM}$ & $28-500 \mathrm{nM}$ & 5 \\
This work & + & Serum & + & $0.18 \mu \mathrm{M}$ & $0.5-10 \mu \mathrm{M}$ & \\
\hline
\end{tabular}

The label "+++" means time-consuming or expensive, "++" means acceptable, "+" means time-saving or cheap.

Table S2. Selection protocol of a successful MTX C-SELEX experiment.

\begin{tabular}{llll}
\hline Rounds & Library $(\mathrm{pmol})$ & MTX concentration $(\mu \mathrm{M})$ & Folic acid concentration $(\mu \mathrm{M})$ \\
\hline 1 & 1000 & 100 & $/$ \\
$2-4$ & 150 & 50 & 100 \\
$5-8$ & 100 & 20 & 100 \\
$9-11$ & 50 & 10 & 150 \\
$12-14$ & 50 & 5 & 150 \\
$15-17$ & 30 & 5 & 200 \\
18 & 20 & 5 & 200 \\
\hline
\end{tabular}

Table S3. DNA sequences used in C-SELEX.

\begin{tabular}{ll}
\hline Name & Sequences \\
\hline Library & GGAGGCTCTCGGGACGAC \\
& NNNNNNNNNNNNNNNNNNNNNNNNNNNNNN \\
& GTCGTCCCGATGCTGCAATCGTAA \\
Forward primer & GGAGGCTCTCGGGACGAC \\
Reverse primer & Biotin-TTACGATTGCAGCATCGGGACGAC \\
Capture & TCCCGAGAGCCTCCTTTT-Biotin \\
\hline
\end{tabular}


Table S4. Sequencing results of a final library pool from MTX aptamer selection.

GGAGGCTCTCGGGACGACACAACGAGGGTTGGTTATGCACCCGACTGCGTCGTCCCGATGCTGCAATCGTAA

GGAGGCTCTCGGGACGACGGACGCGGGATGTTTGGGGGACCCACGTTTGTCGTCCCGATGCTGCAATCGTAA

GGAGGCTCTCGGGACGACGACGGGGCGAACGAACCGGACACACCACGAGTCGTCCCGATGCTGCAATCGTAA

Table S5. DNA sequences used in ITC.

\begin{tabular}{ll}
\hline Name & Sequences \\
\hline HMX72 & GGAGGCTCTCGGGACGACGGACGCGGGATGTTTGGGGGACCCACGTTTGTCGTCCCGATGCTGCAATCGTAA \\
HMX38T & CGACGGACGCGGGATGTTTGGGGGACCCACGTTTGTCG \\
HMX38 & GGGCGAACGCGGGATGTTTGGGGGACCCACGTTCGCCC \\
HMX32 & CGAACGCGGGATGTTTGGGGGACCCACGTTCG \\
HMX24 & CGCGGGATGTTTGGGGGACCCAC \\
G12T & GGGCGAACGCGTGATGTTTGGGGGACCCACGTTCGCCC \\
G16T & GGGCGAACGCGGGATTTTTGGGGGACCCACGTTCGCCC \\
SLAT & GGGCGAACGCGGGATGTTTGAGGGACTCACGTTCGCCC \\
\hline
\end{tabular}


Table S6. DNA sequences of intramolecular strand-displacement fluorescence sensors.

\begin{tabular}{|c|c|}
\hline Name & Sequences \\
\hline \multirow[t]{3}{*}{ HMX38-10T } & FAM- \\
\hline & CTCTCGGGCGAACGCGGGATGTTTGGGGGACCCACGTTCGCCCTTTTTTTTTTCGTTCGCCC \\
\hline & GAGAG-BHQ \\
\hline \multirow{3}{*}{ HMX38-15T } & FAM- \\
\hline & CTCTCGGGCGAACGCGGGATGTTTGGGGGACCCACGTTCGCCCTTTTTTTTTTTTTTTCGTT \\
\hline & CGCCCGAGAG-BHQ \\
\hline \multirow{3}{*}{ HMX38-20T } & FAM- \\
\hline & CTCTCGGGCGAACGCGGGATGTTTGGGGGACCCACGTTCGCCCTTTTTTTTTTTTTTTTTTTT \\
\hline & CGTTCGCCCGAGAG-BHQ \\
\hline \multirow{3}{*}{ HMX38-25T } & FAM- \\
\hline & CTCTCGGGCGAACGCGGGATGTTTGGGGGACCCACGTTCGCCCTTTTTTTTTTTTTTTTTTTT \\
\hline & TTTTTCGTTCGCCCGAGAG-BHQ \\
\hline \multirow{2}{*}{ HMX32-10T } & FAM- \\
\hline & CTCTCCGAACGCGGGATGTTTGGGGGACCCACGTTCGTTTTTTTTTTCGTTCGGAGAG-BHQ \\
\hline \multirow[t]{3}{*}{ HMX32-15T } & FAM- \\
\hline & CTCTCCGAACGCGGGATGTTTGGGGGACCCACGTTCGTTTTTTTTTTTTTTTCGTTCGGAGA \\
\hline & G-BHQ \\
\hline \multirow[t]{3}{*}{ HMX32-20T } & FAM- \\
\hline & CTCTCCGAACGCGGGATGTTTGGGGGACCCACGTTCGTTTTTTTTTTTTTTTTTTTTCGTTCG \\
\hline & GAGAG-BHQ \\
\hline \multirow[t]{3}{*}{ HMX32-25T } & FAM- \\
\hline & CTCTCCGAACGCGGGATGTTTGGGGGACCCACGTTCGTTTTTTTTTTTTTTTTTTTTTTTTTC \\
\hline & GTTCGGAGAG-BHQ \\
\hline HMX28-10T & FAM-CTCGACGCGGGATGTTTGGGGGACCCACGTCTTTTTTTTTTCGTCGAG-BHQ \\
\hline HMX28-15T & FAM-CTCGACGCGGGATGTTTGGGGGACCCACGTCTTTTTTTTTTTTTTTCGTCGAG-BHQ \\
\hline \multirow[t]{2}{*}{ HMX28-20T } & FAM-CTCGACGCGGGATGTTTGGGGGACCCACGTCTTTTTTTTTTTTTTTTTTTTCGTCGAG- \\
\hline & BHQ \\
\hline \multirow[t]{3}{*}{ HMX28-25T } & FAM- \\
\hline & CTCGACGCGGGATGTTTGGGGGACCCACGTCTTTTTTTTTTTTTTTTTTTTTTTTTCGTCGAG- \\
\hline & BHQ \\
\hline
\end{tabular}


Table S7. DNA sequences of intermolecular strand-displacement fluorescence sensors.

\begin{tabular}{ll}
\hline HMX72 sensor & FAM-GGAGGCTCTCGGGACGAC \\
& GGACGCGGGATGTTTGGGGGACCCACGTTT \\
& GTCGTCCCGATGCTGCAATCGTAA \\
Complementary sequence of HMX72 sensor & TCCCGAGAGCCTCC-BHQ \\
HMX38 sensor & FAM/Cy5- \\
& CTCTCGGGCGAACGCGGGATGTTTGGGGGACCCACGTTCGCCC \\
Complementary sequence of HMX38 sensor & CGTTCGCCCGAGAG-BHQ/BHQ3 \\
HMX32 sensor & FAM- \\
& CTCTCCGAACGCGGGATGTTTGGGGGACCCACGTTCG \\
Complementary sequence of HMX32 sensor & CGTTCGGAGAG-BHQ \\
G12T sensor & FAM- \\
Complementary sequence of G12T sensor & CTCTCGGGCGAACGCGTGATGTTTGGGGGACCCACGTTCGCCC \\
G16T sensor & FAM- \\
Complementary sequence of G16T sensor & CTCTCGGGCGAACGCGGGATTTTTGGGGGACCCACGTTCGCCC \\
SLAT sensor & FAM- \\
Complementary sequence of SLAT sensor & CTCTCGGGCGAACGCGGGATGTTTGAGGGACTCACGTTCGCCC \\
\hline
\end{tabular}

\section{Reference:}

1. Begas, E.; Papandreou, C.; Tsakalof, A.; Daliani, D.; Papatsibas, G.; Asprodini, E., Simple and Reliable HPLC Method for the Monitoring of Methotrexate in Osteosarcoma Patients. Journal of Chromatographic Science 2014, 52 (7), 590-595. 2. den Boer, E.; Koch, B. C. P.; Huisman, R.; de Jonge, R., Using Fluorescence Polarization Immunoassay for Determination of Erythrocyte Methotrexate Polyglutamates, a Quick and Easy Test? Therapeutic drug monitoring 2014, 36 (6).

3. Sonemoto, E.; Kono, N.; Ikeda, R.; Wada, M.; Ueki, Y.; Nakashima, K., Practical determination of methotrexate in serum of rheumatic patients by LC-MS/MS. Biomedical Chromatography 2012, 26 (11), 1297-1300.

4. Shi, X.; Gao, H.; Li, Z.; Li, J.; Liu, Y.; Li, L.; Zhang, Q., Modified enzyme multiplied immunoassay technique of methotrexate assay to improve sensitivity and reduce cost. BMC pharmacology \& toxicology 2019, 20 (1), 3.

5. Zhao, S. S.; Bukar, N.; Toulouse, J. L.; Pelechacz, D.; Robitaille, R.; Pelletier, J. N.; Masson, J. F., Miniature multi-channel SPR instrument for methotrexate monitoring in clinical samples. Biosens Bioelectron 2015, 64, 664-70. 